\title{
A GREEN'S FUNCTION FOR A CONVERTIBLE BOND USING THE VASICEK MODEL
}

\author{
R. MALLIER AND A. S. DEAKIN
}

Received 13 March 2002

We consider a convertible security where the underlying stock price obeys a lognormal random walk and the risk-free rate is given by the Vasicek model. Using a Laplace transform in time and a Mellin transform in the stock price, we derive a Green's function solution for the value of the convertible bond.

\section{Introduction}

A convertible bond is defined to be (e.g., Jorion [10]) a bond issued by a corporation that can be converted into the equity of that corporation at certain times using a predetermined exchange ratio. This entails the creation of new shares issued by the corporation if and when conversion occurs, and the existing shares are diluted by the creation of the new shares. The option to convert is solely at the discretion of the bond holder who will do so only if it is beneficial.

Typically, firms issue convertible bonds because they offer a lower interest cost and less restrictive covenants than a nonconvertible bond, but the drawback is that the issuer will be confronted with capital structure uncertainty. Convertible bonds are often subordinated debentures, and because of this, the bond rating agencies have usually rated convertibles one class below that of a straight debenture (Dialynas et al. [7]), and typically issuing convertibles will not affect a company's rating. In return for a reduced yield, an investor will receive a security with considerable upside potential along with downside protection.

Conceptually, the behavior of a convertible bond can be segmented into four regions (e.g., Dialynas et al. [7]). In the late 1990s, most new 
issue convertibles were balanced converts, with around a $25 \%$ conversion premium, where the conversion premium is the excess an investor would pay to acquire the stock by buying the convertible and immediately converting rather than buying the stock itself. Typically, the price of a balanced convert responds materially to changes in both the underlying stock price and the spot interest rate, with a correlation of about $55 \%$ to $80 \%$ with changes in the stock price. A second category is equity substitute converts, where the conversion premium is less than $15 \%$, usually because of rises in the price of the underlying. Typically, the price of an equity substitute responds much more to changes in the stock price than to interest rate changes. A third category is busted converts, where the underlying stock price has declined so significantly that the conversion option is worth very little and the value of the convertible approaches that of an otherwise identical nonconvertible bond. A fourth category is distressed converts, which are busted converts where the stock price has fallen so much that there is a significant chance of bankruptcy.

As of 2000, the market value of convertible securities outstanding globally was in excess of $\$ 400$ billion US, with approximately $\$ 200$ billion in the USA alone (Dialynas et al. [7]). Given the size of the market for these securities, the pricing of convertible bonds is obviously an important problem. Traditionally, convertibles were valued based on the premise that buying a convertible is equivalent to buying the stock at a premium and recouping that premium from the coupons on the convertible, and the payback period is the time taken to recover the premium. More recently, however, contingent claims analysis has been used to value convertibles, which is the approach taken in the present study, and this approach dates back to the work of Ingersoll [9] and Brennan and Schwartz [3]. Brennan and Schwartz originally used the firm value as the underlying variable, and later (Brennan and Schwartz [4]) extended their analysis to include stochastic interest rates and also to include the value of the stock rather than that of the firm (McConnell and Schwartz [11]). Almost all of this earlier work led to a numerical rather than an analytical solution of the underlying equations for the value of a convertible bond, typically using binomial trees; by contrast, the present work in entirely analytical.

In our analysis, we consider a convertible bond, whose value depends on both the price of the underlying stock, which is assumed to obey a lognormal random walk with constant volatility, as in the Black-Scholes option pricing model, and on the interest rate, which is assumed to follow a random walk given by the Vasicek [13] model. We will say more about this interest rate model and its advantages and disadvantages in Section 3. By constructing a risk-free portfolio, it is possible to go from the stochastic differential equations for the stock price and the spot rate 
to a PDE for the value of the convertible (e.g., Wilmott [14]), and that PDE is the starting point for our analysis in the next section. In our analysis, we consider this PDE, and by using a double integral transform, specifically a Laplace transform in time and a Mellin transform with respect to the asset price, we can solve the PDE by transforming it into an ODE and then finding the inverse transforms of the solution. By doing this, we arrive at a Green's function for the price of a convertible.

\section{Analysis}

In this section, we discuss the value $V(S, r, t)$ of a convertible bond. We assume that the asset price $S$ obeys a lognormal random walk

$$
d S=\mu S d t+\sigma S d X_{1}
$$

where $\sigma$ is the volatility of the stock price and $\mu$ is the drift, while the interest rate $r$ obeys

$$
d r=u(r, t) d t+w(r, t) d X_{2}
$$

where $d X_{1}$ and $d X_{2}$ are both normally distributed with zero mean and variance $d t$ and may be correlated, with

$$
E\left[d X_{1} d X_{2}\right]=\rho d t
$$

and $-1 \leq \rho(r, S, t) \leq 1$. Equations (2.1) and (2.2) constitute stochastic differential equations for the underlying stock price and the spot interest rate, respectively. Constructing a risk-free portfolio leads to the following PDE for $V$ :

$$
\frac{\partial V}{\partial t}+\frac{1}{2} \sigma^{2} S^{2} \frac{\partial^{2} V}{\partial S^{2}}+\rho \sigma S w \frac{\partial^{2} V}{\partial S \partial r}+\frac{1}{2} w^{2} \frac{\partial^{2} V}{\partial r^{2}}+r S \frac{\partial V}{\partial S}+(u-\lambda w) \frac{\partial V}{\partial r}-r V=0
$$

the derivation of which is discussed in, for example, Wilmott [14, Chapter 36]. This equation is typically valid for $t \leq T$, where $T$ is the time at which the bond is repaid. In $(2.4), \lambda(r, S, t)$ is the market price of interest rate risk and $u-\lambda w$ is the risk adjusted drift. Many of the popular onefactor interest rate models are special cases of the general affine model for which $u-\lambda w=a(t)-b(t) r$ and $w=(c(t) r-d(t))^{1 / 2}$. Two of these special cases are the Cox-Ingersoll-Ross (CIR) model (Cox et al. [5, 6]) with $u-\lambda w=a-b r$ and $w=c r^{1 / 2}$, where $a, b$, and $c$ are constants, as opposed to functions of $t$ in the general affine model, and the Vasicek 
model, with $u-\lambda w=a-b r$ and $w=c$. The Vasicek model allows interest rates to become negative. Several other special cases of the general affine model are listed in Wilmott [14, Chapter 38]. If we specialize to either the CIR or the Vasicek model, and further make the transformation $t=T-\tau$, so that $\tau$ is the remaining life of the bond, the PDE above becomes

$$
\begin{aligned}
\frac{\partial V}{\partial \tau}= & \frac{1}{2} \sigma^{2} S^{2} \frac{\partial^{2} V}{\partial S^{2}}+\rho \sigma c r^{m} S \frac{\partial^{2} V}{\partial S \partial r}+\frac{1}{2} c^{2} r^{2 m} \frac{\partial^{2} V}{\partial r^{2}} \\
& +r S \frac{\partial V}{\partial S}+(a-b r) \frac{\partial V}{\partial r}-r V,
\end{aligned}
$$

where $m=0$ for the Vasicek model and $1 / 2$ for CIR. We will suppose the pay-off at expiry, $t=T$ or $\tau=0$, is $V_{0}(S, r)=V(S, r, 0)$. For a Europeanstyle convertible discount bond, with pay-off 1 at expiry, which can be converted for one unit of stock of value $S$ at expiry, the effective payoff at expiry is $V_{0}(S, r)=\max (S, 1)$. To analyze the PDE (2.5), we take a Laplace transform with respect to time $\tau$,

$$
\mathcal{L}[f(\tau)]=\int_{0}^{\infty} e^{-z \tau} f(\tau) d \tau,
$$

and a Mellin transform with respect to the price of the underlying $S$,

$$
\mathcal{M}[f(S)]=\int_{0}^{\infty} S^{p-1} f(S) d S,
$$

so that

$$
\mho(p, r, z)=\mathcal{M}[\mathcal{L}(V(S, r, \tau))]=\int_{0}^{\infty} S^{p-1}\left[\int_{0}^{\infty} e^{-z \tau} V(S, r, \tau) d \tau\right] d S .
$$

Noting that

$$
\begin{aligned}
\mathcal{M}\left[S f^{\prime}(S)\right] & =-p \mathcal{M}[f(S)], \\
\mathcal{M}\left[S^{2} f^{\prime \prime}(S)\right] & =p(p+1) \mathcal{M}[f(S)], \\
\mathcal{L}\left[f^{\prime}(\tau)\right] & =z \mathcal{L}[f(S)]-f(0),
\end{aligned}
$$

we arrive at the following ODE for the transform of the option price:

$$
\begin{aligned}
\frac{1}{2} c^{2} r^{2 m} & \frac{\partial^{2} \mho}{\partial r^{2}}+\left(a-\rho \sigma c p r^{m}-b r\right) \frac{\partial \mho}{\partial r} \\
& +\left[\left(\frac{1}{2} \sigma^{2} p-r\right)(1+p)-z\right] \mho+\mathcal{M}\left[V_{0}(S, r)\right]=0 .
\end{aligned}
$$


In order to value the bond using the method considered here, it is necessary to first solve the ODE (2.10) and then invert both the Mellin and Laplace transforms. For the CIR model, with $m=1 / 2$, (2.10) becomes

$$
\begin{aligned}
& \frac{1}{2} c^{2} r \frac{\partial^{2} \mho}{\partial r^{2}}+\left(a-\rho \sigma c p r^{1 / 2}-b r\right) \frac{\partial \mho}{\partial r} \\
& +\left[\left(\frac{1}{2} \sigma^{2} p-r\right)(1+p)-z\right] \mho+\mathcal{M}\left[V_{0}(S, r)\right]=0 ;
\end{aligned}
$$

the presence of the $r^{1 / 2}$ term makes this equation difficult to solve, and we have been unable to find a closed form solution. For the Vasicek model with $m=0$, on the other hand, we have

$$
\begin{aligned}
\frac{1}{2} c^{2} \frac{\partial^{2} \mho}{\partial r^{2}} & +(a-\rho \sigma c p-b r) \frac{\partial \mho}{\partial r} \\
+ & {\left[\left(\frac{1}{2} \sigma^{2} p-r\right)(1+p)-z\right] \mho+\mathcal{M}\left[V_{0}(S, r)\right]=0, }
\end{aligned}
$$

which can be solved in terms of the Kummer functions $M$ and $U$ (Abramowitz and Stegun [1]), also known as confluent hypergeometric functions. Two linearly independent homogeneous solutions to (2.12) are

$$
\begin{array}{r}
\mho_{1}=\left(a b-c^{2}(1+p)-b p c \sigma \rho-b^{2} r\right) \\
\times \exp \left[-\frac{r(1+p)}{b}-\frac{\left(a b-c^{2}(1+p)-b p c \sigma \rho\right)^{2}}{2 c^{2} b^{3}}\right] \\
\times M\left[\frac{(1+p)\left(2 a b-c^{2}(1+p)-b^{2} \sigma^{2} p-2 b p c \sigma \rho\right)}{4 b^{3}}\right. \\
\left.+\frac{b+z}{2 b}, \frac{3}{2}, \frac{\left(a b-c^{2}(1+p)-b p c \sigma \rho-b^{2} r\right)^{2}}{c^{2} b^{3}}\right], \\
\mho_{2}=\left(a b-c^{2}(1+p)-b p c \sigma \rho-b^{2} r\right) \\
\times \exp \left[-\frac{r(1+p)}{b}-\frac{\left(a b-c^{2}(1+p)-b p c \sigma \rho\right)^{2}}{2 c^{2} b^{3}}\right] \\
\times U\left[\frac{(1+p)\left(2 a b-c^{2}(1+p)-b^{2} \sigma^{2} p-2 b p c \sigma \rho\right)}{4 b^{3}}\right], \\
\left.+\frac{b+z}{2 b}, \frac{3}{2}, \frac{\left(a b-c^{2}(1+p)-b p c \sigma \rho-b^{2} r\right)^{2}}{c^{2} b^{3}}\right],
\end{array}
$$


and the Wronskian of these two solutions is

$$
\begin{aligned}
W= & \mho_{1} \mho_{2}^{\prime}-\mho_{1}^{\prime} \mho_{2} \\
= & -\left[\Gamma\left(\frac{(1+p)\left(2 a b-c^{2}(1+p)-b^{2} \sigma^{2} p-2 b p c \sigma \rho\right)}{4 b^{3}}+\frac{b+z}{2 b}\right)\right]^{-1} \\
& \times c b^{7 / 2} \sqrt{\pi} \exp \left[\frac{b r^{2}-2(a-p c \sigma \rho) r}{c^{2}}\right],
\end{aligned}
$$

where we have used the fact that $\Gamma(3 / 2)=\sqrt{\pi} / 2$. The general solution to $(2.12)$ using variation of parameters is

$$
\begin{aligned}
\mho^{\prime}= & \frac{2}{c^{2}}\left(\mho_{1}\left[A_{1}+\int^{r} \frac{\mho_{2} \mathcal{M}\left[V_{0}(S, \tilde{r})\right] d \tilde{r}}{\mathcal{W}}\right]+\mho_{2}\left[A_{2}-\int^{r} \frac{\mho_{1} \mathcal{M}\left[V_{0}(S, \tilde{r})\right] d \tilde{r}}{w^{2}}\right]\right) \\
= & -\frac{2}{c^{3} b^{7 / 2} \sqrt{\pi}} \Gamma\left(\frac{(1+p)\left(2 a b-c^{2}(1+p)-b^{2} \sigma^{2} p-2 b p c \sigma \rho\right)}{4 b^{3}}+\frac{b+z}{2 b}\right) \\
& \times\left[\mho_{1}\left(B_{1}+\int^{r} \mho_{2} \mathcal{M}\left[V_{0}(S, \tilde{r})\right] \exp \left[\frac{-b \tilde{r}^{2}+2(a-p c \sigma \rho) \tilde{r}}{c^{2}}\right] d \tilde{r}\right)\right. \\
& \left.+\mho_{2}\left(B_{2}-\int^{r} \mho_{1} \mathcal{M}\left[V_{0}(S, \tilde{r})\right] \exp \left[\frac{-b \tilde{r}^{2}+2(a-p c \sigma \rho) \tilde{r}}{c^{2}}\right] d \tilde{r}\right)\right] .
\end{aligned}
$$

In applying this solution to the problem at hand, we must recall that the spot interest rate $r$ obeys the random walk (2.2), and that for the Vasicek model $r$ can range between $-\infty$ and $\infty$. The value of the convertible bond comes from the expected pay-off as $r$ and the stock price $S$ follow their respective random walks; because of this, we would expect that as the end result of the present study, we can write the price of the bond as a double integral over the possible ranges of both $S$ and $r$, involving both the pay-off for each possible pair of values of $S$ and $r$ and also the probability of hitting those values of $S$ and $r$ from their current values. In addition, we have the boundary condition that $V$ is bounded as $r \rightarrow \infty$. Using the asymptotic behavior of the Kummer functions (Abramowitz and Stegun [1]), this leads us to deduce that 


$$
\begin{aligned}
\mho= & \frac{2}{c^{3} b^{7 / 2} \sqrt{\pi}} \Gamma\left(\frac{(1+p)\left(2 a b-c^{2}(1+p)-b^{2} \sigma^{2} p-2 b p c \sigma \rho\right)}{4 b^{3}}+\frac{b+z}{2 b}\right) \\
& \times\left[\mho_{1} \int_{r}^{\infty} \mho_{2} \mathcal{M}\left[V_{0}(S, \tilde{r})\right] \exp \left[\frac{-b \tilde{r}^{2}+2(a-p c \sigma \rho) \tilde{r}}{c^{2}}\right] d \tilde{r}\right. \\
& \left.+\mho_{2} \int_{-\infty}^{r} \mho_{1} \mathcal{M}\left[V_{0}(S, \tilde{r})\right] \exp \left[\frac{-b \tilde{r}^{2}+2(a-p c \sigma \rho) \tilde{r}}{c^{2}}\right] d \tilde{r}\right] .
\end{aligned}
$$

Having solved (2.12), we must now invert the Laplace and Mellin transforms (2.6) and (2.7). The inverse Laplace transform is defined to be

$$
\mathcal{L}^{-1}[F(z)]=\frac{1}{2 \pi i} \int_{\gamma-i \infty}^{\gamma+i \infty} e^{z \tau} F(z) d z
$$

while the inverse Mellin transform is given by

$$
\mathcal{M}^{-1}[F(p)]=\frac{1}{2 \pi i} \int_{\delta-i \infty}^{\delta+i \infty} S^{-p} F(p) d p
$$

Inverting the Laplace transform first, we must pick the contour so that $\gamma$ lies to the right of all singularities. We can evaluate this integral by closing the contour to the left with a semicircle at infinity, and the value of the contour integral is $2 \pi i$ times the sum of the residues contained inside the loop. Recalling that $\Gamma(c z)$ is single valued and analytic over the entire complex plane, except for simple poles with residue $(-1)^{n} c^{-1} / n$ ! at the points $z=-n / c(n=0,1,2, \ldots)$, we deduce that $\mho$ has simple poles at the points

$$
z=-b(1+2 n)-\frac{(1+p)\left(2 a b-c^{2}(1+p)-b^{2} \sigma^{2} p-2 b p c \sigma \rho\right)}{2 b^{2}},
$$

and it follows that

$$
\begin{aligned}
\mathcal{M}[V]= & \frac{4\left(a b-c^{2}(1+p)-b p c \sigma \rho-b^{2} r\right)}{c^{3} b^{5 / 2} \sqrt{\pi}} \\
& \times \exp \left[-\frac{r(1+p)}{b}-\frac{\left(a b-c^{2}(1+p)-b p c \sigma \rho\right)^{2}}{c^{2} b^{3}}\right] \\
& \times \exp \left(\left[-b-\frac{(1+p)\left(2 a b-c^{2}(1+p)-b^{2} \sigma^{2} p-2 b p c \sigma \rho\right)}{2 b^{2}}\right] \tau\right)
\end{aligned}
$$


A convertible bond

$$
\begin{aligned}
\times \sum_{n=0}^{\infty} \frac{(-1)^{n} e^{-2 n b \tau}}{n !}[ & M\left[-n, \frac{3}{2}, \frac{\left(a b-c^{2}(1+p)-b p c \sigma \rho-b^{2} r\right)^{2}}{c^{2} b^{3}}\right] \\
& \times \int_{r}^{\infty} U\left[-n, \frac{3}{2}, \frac{\left(a b-c^{2}(1+p)-b p c \sigma \rho-b^{2} \tilde{r}\right)^{2}}{c^{2} b^{3}}\right] \\
& \times \mathcal{M}\left[V_{0}(S, \tilde{r})\right]\left(a b-c^{2}(1+p)-b p c \sigma \rho-b^{2} \tilde{r}\right) \\
& \times \exp \left[\frac{-b r^{2}+2(a-p c \sigma \rho) \tilde{r}}{c^{2}}-\frac{\tilde{r}(1+p)}{b}\right] d \tilde{r} \\
& +U\left[-n, \frac{3}{2}, \frac{\left(a b-c^{2}(1+p)-b p c \sigma \rho-b^{2} r\right)^{2}}{c^{2} b^{3}}\right] \\
& \times \int_{-\infty}^{r} M\left[-n, \frac{3}{2}, \frac{\left(a b-c^{2}(1+p)-b p c \sigma \rho-b^{2} \tilde{r}\right)^{2}}{c^{2} b^{3}}\right] \\
& \times \mathbb{M}\left[V_{0}(S, \tilde{r})\right]\left(a b-c^{2}(1+p)-b p c \sigma \rho-b^{2} \tilde{r}\right) \\
& \left.\times \exp \left[\frac{-b \tilde{r}^{2}+2(a-p c \sigma \rho) \tilde{r}}{c^{2}}-\frac{\tilde{r}(1+p)}{b}\right] d \tilde{r}\right] .
\end{aligned}
$$

At first glance, this expression (2.20) appears to be an extremely complication function of the Mellin transform variable $p$, because of the continued presence of the Kummer functions. Fortunately, we can simplify it considerably using several identities for special functions. Firstly, we can rewrite (2.20) in terms of Laguerre polynomials using the relations (Abramowitz and Stegun [1])

$$
\begin{aligned}
& M(-n, \hat{k}, \hat{r})=\frac{n ! \Gamma(\hat{k})}{\Gamma(\hat{k}+n)} L_{n}^{\hat{k}-1}(\hat{r}), \\
& U(-n, \hat{k}, \hat{r})=(-1)^{n} n ! L_{n}^{\hat{k}-1}(\hat{r}),
\end{aligned}
$$

which leads to a greatly simplified expression,

$$
\begin{aligned}
\mathcal{M}[V]= & \frac{4\left(a b-c^{2}(1+p)-b p c \sigma \rho-b^{2} r\right)}{c^{3} b^{5 / 2} \sqrt{\pi}} \\
& \times \exp \left[-\frac{r(1+p)}{b}-\frac{\left(a b-c^{2}(1+p)-b p c \sigma \rho\right)^{2}}{c^{2} b^{3}}\right] \\
& \times \exp \left(\left[-b-\frac{(1+p)\left(2 a b-c^{2}(1+p)-b^{2} \sigma^{2} p-2 b p c \sigma \rho\right)}{2 b^{2}}\right] \tau\right)
\end{aligned}
$$




$$
\begin{aligned}
& \times \sum_{n=0}^{\infty} \frac{n ! \Gamma(3 / 2)}{\Gamma(3 / 2+n)} L_{n}^{1 / 2}\left[\frac{\left(a b-c^{2}(1+p)-b p c \sigma \rho-b^{2} r\right)^{2}}{c^{2} b^{3}}\right] e^{-2 n b \tau} \\
& \times \int_{-\infty}^{\infty} L_{n}^{1 / 2}\left[\frac{\left(a b-c^{2}(1+p)-b p c \sigma \rho-b^{2} \tilde{r}\right)^{2}}{c^{2} b^{3}}\right] \\
& \times \mathcal{M}\left[V_{0}(S, \tilde{r})\right]\left(a b-c^{2}(1+p)-b p c \sigma \rho-b^{2} \tilde{r}\right) \\
& \times \exp \left[\frac{-b \tilde{r}^{2}+2(a-p c \sigma \rho) \tilde{r}}{c^{2}}-\frac{\tilde{r}(1+p)}{b}\right] d \tilde{r} \\
& =\frac{2\left(a b-c^{2}(1+p)-b p c \sigma \rho-b^{2} r\right)}{c^{3} b^{5 / 2}} \\
& \times \exp \left[-\frac{r(1+p)}{b}-\frac{\left(a b-c^{2}(1+p)-b p c \sigma \rho\right)^{2}}{c^{2} b^{3}}\right] \\
& \times \exp \left(\left[-b-\frac{(1+p)\left(2 a b-c^{2}(1+p)-b^{2} \sigma^{2} p-2 b p c \sigma \rho\right)}{2 b^{2}}\right] \tau\right) \\
& \times \int_{-\infty}^{\infty} \mathcal{M}\left[V_{0}(S, \tilde{r})\right] \\
& \times\left(\sum_{n=0}^{\infty} \frac{n ! e^{-2 n b \tau}}{\Gamma(3 / 2+n)} L_{n}^{1 / 2}\left[\frac{\left(a b-c^{2}(1+p)-b p c \sigma \rho-b^{2} r\right)^{2}}{c^{2} b^{3}}\right]\right. \\
& \left.\times L_{n}^{1 / 2}\left[\frac{\left(a b-c^{2}(1+p)-b p c \sigma \rho-b^{2} \tilde{r}\right)^{2}}{c^{2} b^{3}}\right]\right) \\
& \times\left(a b-c^{2}(1+p)-b p c \sigma \rho-b^{2} \tilde{r}\right) \\
& \times \exp \left[\frac{-b \tilde{r}^{2}+2(a-p c \sigma \rho) \tilde{r}}{c^{2}}-\frac{\tilde{r}(1+p)}{b}\right] d \tilde{r} \text {. }
\end{aligned}
$$

One important point to note is that the two separate integrals from $\tilde{r}=$ $-\infty$ to $r$ and from $\tilde{r}=r$ to $\infty$ have now been combined into a single integral over the range $-\infty \leq \tilde{r} \leq \infty$. This expression can be simplified still further. We know (Gradšteĭn and Ryžik [8]) that

$$
\begin{aligned}
& \sum_{n=0}^{\infty} \frac{n ! z^{n}}{\Gamma(n+\alpha+1)} L_{n}^{\alpha}(x) L_{n}^{\alpha}(y) \\
& \quad=\frac{(x y z)^{-\alpha / 2}}{1-z} \exp \left[-\frac{z(x+y)}{1-z}\right] I_{\alpha}\left[\frac{2 \sqrt{x y z}}{1-z}\right],
\end{aligned}
$$


228 A convertible bond

so with $\alpha=1 / 2$ and $z=e^{-2 b \tau}$, we have

$$
\begin{aligned}
& \sum_{n=0}^{\infty} \frac{n ! e^{-2 n b \tau}}{\Gamma(3 / 2+n)} L_{n}^{1 / 2}(x) L_{n}^{1 / 2}(y) \\
& \quad=(x y)^{-1 / 4} \frac{e^{b \tau / 2}}{1-e^{-2 b \tau}} \exp \left[-\frac{e^{-2 b \tau}}{1-e^{-2 b \tau}}(x+y)\right] I_{1 / 2}\left[2 \sqrt{x y} \frac{e^{-b \tau}}{1-e^{-2 b \tau}}\right] .
\end{aligned}
$$

Since $I_{1 / 2}(x)=\sqrt{2 /(\pi x)} \sinh x$, we have

$$
\begin{aligned}
\sum_{n=0}^{\infty} \frac{n ! e^{-2 n b \tau}}{\Gamma(3 / 2+n)} L_{n}^{1 / 2}(x) L_{n}^{1 / 2}(y) \\
=(x y)^{-1 / 2} \pi^{-1 / 2} \frac{e^{b \tau}}{\sqrt{1-e^{-2 b \tau}}} \exp \left[-\frac{e^{-2 b \tau}}{1-e^{-2 b \tau}}(x+y)\right] \\
\quad \times \sinh \left[2 \sqrt{x y} \frac{e^{-b \tau}}{1-e^{-2 b \tau}}\right] .
\end{aligned}
$$

With this, our expression (2.22) becomes

$$
\begin{aligned}
& \mathcal{M}[V]=\frac{2 \sqrt{b}}{c \sqrt{\pi} \sqrt{1-e^{-2 b \tau}}} \exp \left[-\frac{r(1+p)}{b}-\frac{\left(a b-c^{2}(1+p)-b p c \sigma \rho\right)^{2}}{c^{2} b^{3}}\right] \\
& \times \exp \left(\left[-\frac{(1+p)\left(2 a b-c^{2}(1+p)-b^{2} \sigma^{2} p-2 b p c \sigma \rho\right)}{2 b^{2}}\right] \tau\right) \\
& \times \int_{-\infty}^{\infty} \mathcal{M}\left[V_{0}(S, \tilde{r})\right] \\
& \times \exp \left[-\frac{e^{-2 b \tau}\left(a b-c^{2}(1+p)-b p c \sigma \rho-b^{2} r\right)^{2}}{c^{2} b^{3}\left(1-e^{-2 b \tau}\right)}\right. \\
& \left.+\frac{e^{-2 b \tau}\left(a b-c^{2}(1+p)-b p c \sigma \rho-b^{2} \tilde{r}\right)^{2}}{c^{2} b^{3}\left(1-e^{-2 b \tau}\right)}\right] \\
& \times \sinh \left[\frac{2 e^{-b \tau}}{c^{2} b^{3}\left(1-e^{-2 b \tau}\right)}\left(a b-c^{2}(1+p)-b p c \sigma \rho-b^{2} r\right)\right. \\
& \left.\times\left(a b-c^{2}(1+p)-b p c \sigma \rho-b^{2} \tilde{r}\right)\right] \\
& \times \exp \left[\frac{-b \tilde{r}^{2}+2(a-p c \sigma \rho) \tilde{r}}{c^{2}}-\frac{\tilde{r}(1+p)}{b}\right] d \tilde{r} \text {. }
\end{aligned}
$$


We can cast this in the form

$$
\mathcal{M}[V]=\frac{\sqrt{b}}{c \sqrt{\pi} \sqrt{1-e^{-2 b \tau}}} \int_{-\infty}^{\infty} \mathcal{M}\left[V_{0}(S, \tilde{r})\right]\left[e^{\alpha_{+} p^{2}+\beta_{+} p+\gamma_{+}}-e^{\alpha_{-} p^{2}+\beta_{-} p+\gamma_{-}}\right] d \tilde{r},
$$

where $\alpha_{ \pm}, \beta_{ \pm}$, and $\gamma_{ \pm}$are functions of $r, \tilde{r}$, and $\tau$, and are given by

$$
\begin{aligned}
\alpha_{ \pm}= & \frac{ \pm\left(c+\rho \sigma b^{2}\right)^{2}-2 \cosh b \tau\left(\rho \sigma b+c^{2}\right)}{2 b^{3} \sinh b \tau}+\frac{\tau\left(\sigma^{2} b^{2}+c^{2}+2 c \rho \sigma b\right)}{2 b^{2}}, \\
\beta_{ \pm}= & \frac{ \pm\left(c+\rho \sigma b^{2}\right)\left(2 c^{2}-2 b^{2} a+b^{2}(r+\tilde{r})\right)-4 \cosh b \tau(\rho \sigma b+c)\left(c^{2}-b a\right)}{2 b^{3} c \sinh b \tau} \\
& +\frac{\left(2 c \rho \sigma b+\sigma^{2} b^{2}+2 c^{2}-2 b a\right) \tau}{2 b^{2}}-\frac{\rho \sigma b\left(\tilde{r} e^{b \tau}+r e^{-b \tau}\right)+c(r+\tilde{r}) \cosh b \tau}{b c \sinh b \tau}, \\
\gamma_{ \pm}= & \pm\left(b^{2} \tilde{r}-b^{2} a+c^{2}\right)\left(b^{2} r-b^{2} a+c^{2}\right) \\
2 b^{3} c^{2} \sinh b \tau & \frac{\left(c^{2}-2 b a\right) \tau}{2 b^{2}} \\
& -\frac{b\left(r^{2} e^{-b \tau}+\tilde{r}^{2} e^{b \tau}\right)}{2 c^{2} \sinh b \tau}+\frac{a\left(r e^{-b \tau}+\tilde{r} e^{b \tau}\right)}{c^{2} \sinh b \tau} \\
& -\frac{\left(c^{2} b^{2}(r+\tilde{r})+\left(a b-c^{2}\right)^{2}\right) \cosh b \tau}{b^{3} c^{2} \sinh b \tau}
\end{aligned}
$$

where we take the "+" signs in (2.28) for $\alpha_{+}, \beta_{+}$, and $\gamma_{+}$, and the "-" signs for $\alpha_{-}, \beta_{-}$, and $\gamma_{-}$. The reason for writing $\mathcal{M}[V]$ in the form (2.27) is that the Mellin transform can then be inverted using tables (Polyanin and Manzhirov [12]), using the inverse transform

$$
\mathcal{M}^{-1}\left[e^{\alpha p^{2}+\beta p+\gamma}\right]=\frac{1}{2 \sqrt{\pi \alpha}} \exp \left[\gamma-\frac{(\beta-\log S)^{2}}{4 \alpha}\right]
$$

and the relation

$$
\mathcal{M}^{-1}\left[F_{1}(p) F_{2}(p)\right]=\int_{0}^{\infty} f_{1}(S / \tilde{S}) f_{2}(\tilde{S}) \tilde{S}^{-1} d \tilde{S}
$$

which tells us that

$$
\begin{aligned}
& \mathcal{M}^{-1}\left(\int_{-\infty}^{\infty} \mathcal{M}\left[V_{0}(S, \tilde{r})\right] e^{\alpha p^{2}+\beta p+\gamma} d \tilde{r}\right) \\
& \quad=\int_{-\infty}^{\infty} \int_{0}^{\infty} \frac{1}{2 \sqrt{\pi \alpha}} \exp \left[\gamma-\frac{(\beta-\log (S / \tilde{S}))^{2}}{4 \alpha}\right] V_{0}(\tilde{S}, \tilde{r}) \tilde{S}^{-1} d \tilde{S} d \tilde{r}
\end{aligned}
$$


It follows that our inverse is

$$
\begin{aligned}
V= & \frac{\sqrt{b}}{\pi c \sqrt{1-e^{-2 b \tau}}} \\
& \times \int_{-\infty}^{\infty} \int_{0}^{\infty}\left(\alpha_{+}^{-1 / 2} \exp \left[\gamma_{+}-\frac{\left(\beta_{+}-\log (S / \tilde{S})\right)^{2}}{4 \alpha_{+}}\right]\right. \\
& \left.\quad-\alpha_{-}^{-1 / 2} \exp \left[\gamma_{-}-\frac{\left(\beta_{-}-\log (S / \tilde{S})\right)^{2}}{4 \alpha_{-}}\right]\right) V_{0}(\tilde{S}, \tilde{r}) \tilde{S}^{-1} d \tilde{S} d \tilde{r}
\end{aligned}
$$

where $\alpha_{ \pm}, \beta_{ \pm}$, and $\gamma_{ \pm}$are given by (2.28). This solution can of course be written in the form

$$
V=\int_{-\infty}^{\infty} \int_{0}^{\infty} G(S, \tilde{S}, r, \tilde{r}, \tau) V_{0}(\tilde{S}, \tilde{r}) d \tilde{S} d \tilde{r}
$$

where

$$
\begin{aligned}
G(S, \tilde{S}, r, \tilde{r}, \tau)=\frac{\tilde{S}^{-1} \sqrt{b}}{\pi c \sqrt{1-e^{-2 b \tau}}}\left(\alpha_{+}^{-1 / 2} \exp \left[\gamma_{+}-\frac{\left(\beta_{+}-\log (S / \tilde{S})\right)^{2}}{4 \alpha_{+}}\right]\right. \\
\left.-\alpha_{-}^{-1 / 2} \exp \left[\gamma_{-}-\frac{\left(\beta_{-}-\log (S / \tilde{S})\right)^{2}}{4 \alpha_{-}}\right]\right),
\end{aligned}
$$

is the Green's function.

\section{Discussion}

The principle result of this paper is the Green's function solution (2.32) for the value of a convertible bond under the dual assumptions that the value of the underlying stock obeys a lognormal random walk and the spot interest rate is given by the Vasicek model. The valuation of convertible bonds is an important problem, not least because the market value of such instruments currently outstanding is several hundred billion dollars. Our end result is of course quite flexible and can be adapted to several situations by the choice of an appropriate pay-off at expiry, $V_{0}(S, r)$. For example, for a European style convertible bond, meaning one that can only be converted at expiry, the pay-off would be $V_{0}(S, r)=\max (S, 1)$ if the conversion ratio was one, and we have assumed that the number of shares outstanding is very large so that we can neglect dilution. Similarly, we can use our solution to price a semi-American (or Bermudan) style convertible bond which can be exercised at a series of discrete 
dates, for example, $t=T, T-\tau_{0}, T-2 \tau_{0}, \ldots$, which might coincide with the coupon dates of the bond, by valuing the bond separately on successive time intervals, typically, the pay-off would be known at $t=T$, the expiration of the bond, and we can use this to arrive at a value for the bond on the interval $T \leq t \leq T-\tau_{0}$ using our solution (2.32), and use this to arrive at the pay-off at time $t=T-\tau_{0}$, which we can use in (2.32) to yield a value of the bond on the interval $T-\tau_{0} \leq t \leq T-2 \tau_{0}$, and so on. As a further extension, most convertibles are also callable at the discretion of the issuing corporation (Jorion [10]), and this call feature could also be included in the pay-off at expiry $V_{0}(S, r)$. Similarly, some convertibles also have embedded put options (Bhattacharya [2]), and these could also be included in $V_{0}(S, r)$.

A few words should also be said about the model used in our study, where we have combined the standard lognormal random walk for the stock price with the Vasicek model for the spot rate. The primary rationale for using the Vasicek model is that it is extremely tractable; it is also mean reverting which is desirable, but has the undesirable property that interest rates can go negative, which is why the integral over $\tilde{r}$ in (2.32) extends from $-\infty$ to $\infty$. Obviously, more realistic models exist, and a similar analysis to ours could in principal be performed for some of those models. However, most of these models are somewhat less tractable than the Vasicek model, for example, for the CIR model mentioned earlier, which many researchers hold to be a more realistic model, we have been unable to date to solve the ODE (2.11) for the transform of the bond price, and unless and until that equation is solved, we cannot proceed with the analysis for the CIR model; by contrast, the corresponding ODE (2.12) for the Vasicek model was comparatively easy to solve, with the solution given by (2.16).

\section{References}

[1] M. Abramowitz and I. A. Stegun, Handbook of Mathematical Functions with Formulas, Graphs, and Mathematical Tables, National Bureau of Standards Applied Mathematics Series, vol. 55, U.S. Government Printing Office, Washington, D.C., 1964.

[2] M. Bhattacharya, Convertible securities and their valuation, Handbook of Fixed Income Securities (F. J. Fabozzi, ed.), McGraw-Hill, New York, 6th ed., 2001, pp. 1127-1171.

[3] M. J. Brennan and E. S. Schwartz, Convertible bonds: valuation and optimal strategies for call and conversion, J. Finance 32 (1977), 1699-1715.

[4] , Analyzing convertible bonds, J. Fin. Quant. Anal. 15 (1980), 907-929.

[5] J. C. Cox, J. E. Ingersoll Jr., and S. A. Ross, An intertemporal general equilibrium model of asset prices, Econometrica 53 (1985), no. 2, 363-384.

[6] A theory of the term structure of interest rates, Econometrica 53 (1985), no. $2,385-407$. 


\section{A convertible bond}

[7] C. P. Dialynas, S. Durn, and J. C. Ritchie, Convertible securities and their investment characteristics, Handbook of Fixed Income Securities (F. J. Fabozzi, ed.), McGraw-Hill, New York, 6th ed., 2001, pp. 1103-1126.

[8] I. S. Gradšteřn and I. M. Ryžik, Tables of Integrals, Sums, Series and Products, Gosudarstv. Izdat. Fiz.-Mat. Lit., Moscow, 1963 (Russian).

[9] J. E. Ingersoll, A contingent claim valuation of convertible securities, J. Financial Economics 4 (1977), 289-322.

[10] P. Jorion, Financial Risk Manager Handbook 2001-2002, John Wiley \& Sons, New York, 2001.

[11] J. J. McConnell and E. S. Schwartz, LYON taming, J. Finance 41 (1986), 561576.

[12] A. D. Polyanin and A. V. Manzhirov, Handbook of Integral Equations, CRC Press, Florida, 1998.

[13] O. A. Vasicek, An equilibrium characterization of the term structure, J. Financial Economics 5 (1977), 177-188.

[14] P. Wilmott, Derivatives. The Theory and Practice of Financial Engineering, John Wiley \& Sons, Chichester, 1998.

R. Mallier: Department of Applied Mathematics, The University of Western Ontario, London, Ontario, Canada N6A 5B7

E-mail address: asdeakin@uwo.ca

A. S. Deakin: Department of Applied Mathematics, The University of Western Ontario, London, Ontario, Canada N6A 5B7 


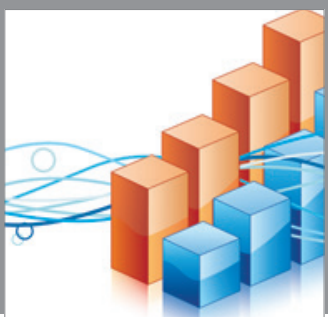

Advances in

Operations Research

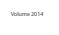

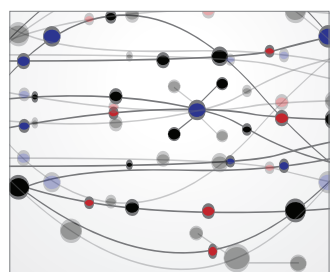

\section{The Scientific} World Journal
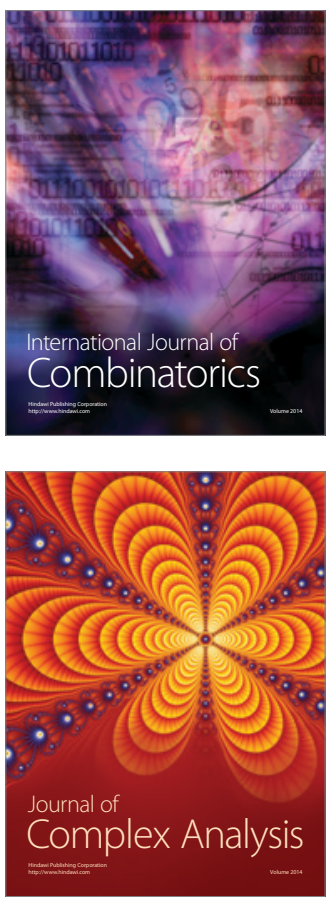

International Journal of

Mathematics and

Mathematical

Sciences
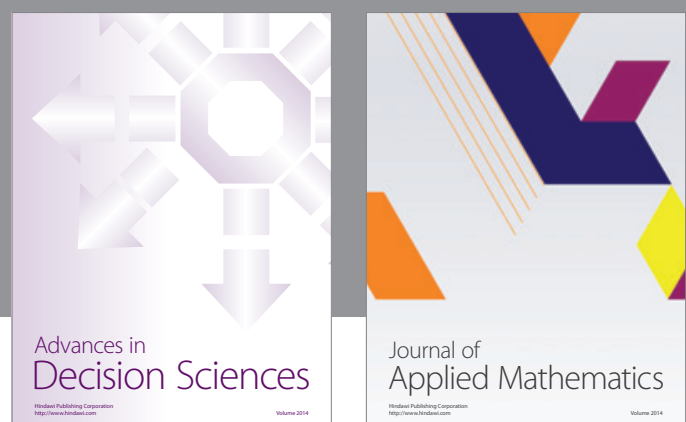

Journal of

Applied Mathematics
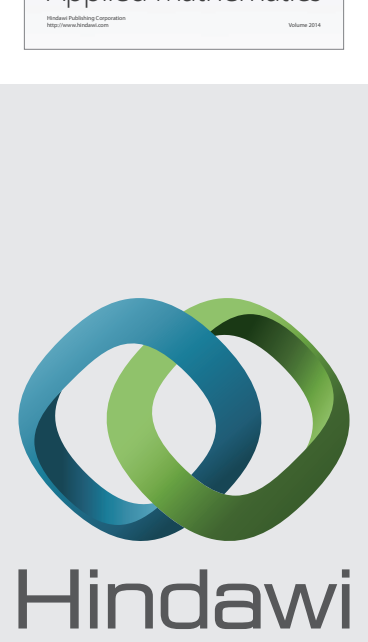

Submit your manuscripts at http://www.hindawi.com
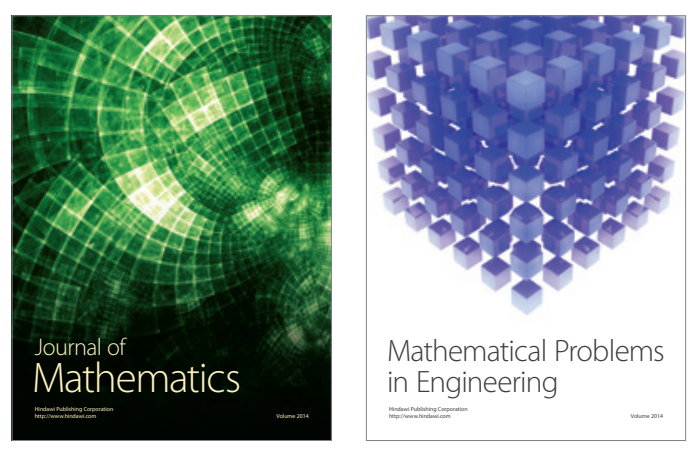

Mathematical Problems in Engineering
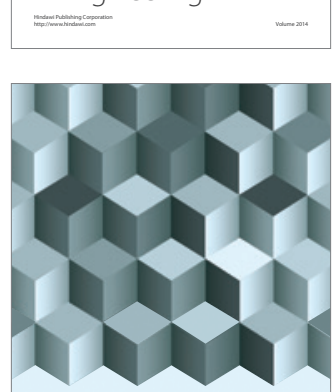

Journal of

Function Spaces
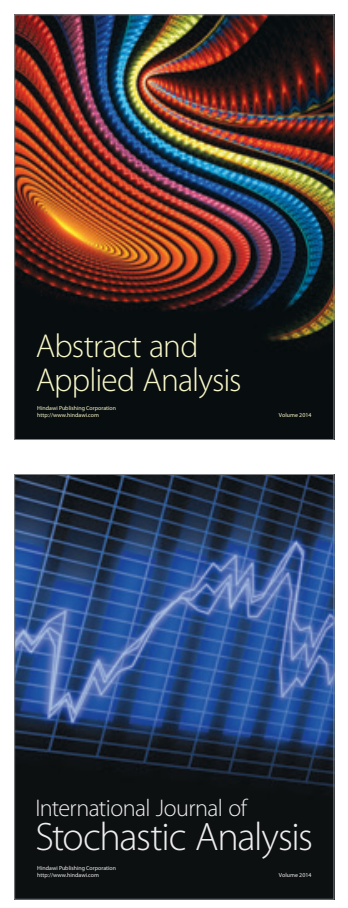

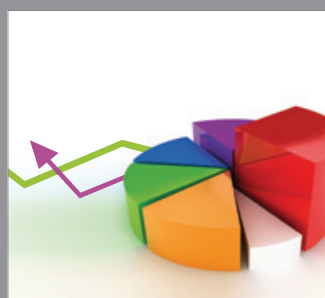

ournal of

Probability and Statistics

Promensencen
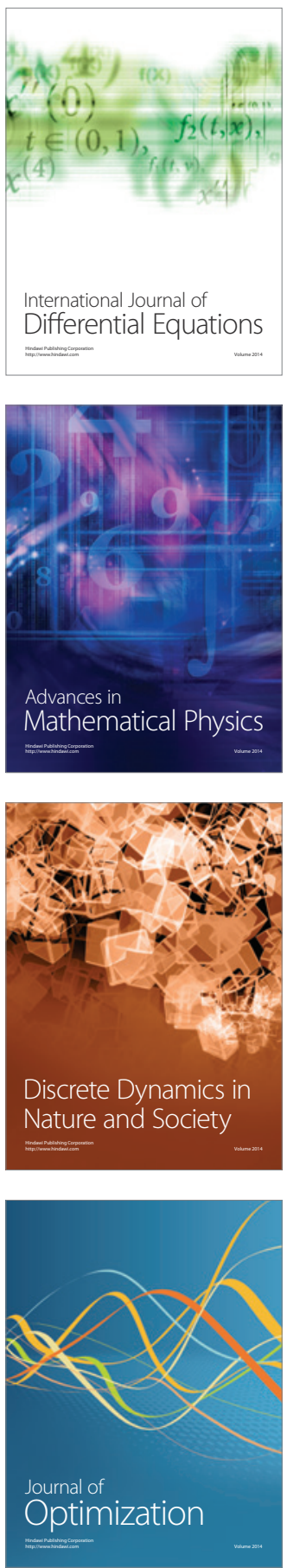